\title{
Traumatic rupture of the thoracic aorta
}

\author{
G. KEEN, R. A. BRADBR O OK, A N D F. M C I N N \\ From the United Bristol Hospitals
}

\begin{abstract}
Seven patients who had traumatic ruptures of the thoracic aorta are reported. Four of these died within a few hours of admission, allowing no opportunity for diagnosis or treatment. However, three survived long enough for elective surgery to be undertaken. A diagnosis of ruptured aorta was missed in one patient (case 2), and the difficulties of diagnosing this condition, even during thoracotomy, are emphasized. The value of serial chest radiography and forward aortography is discussed. Two of these patients underwent successful aortic repair, using left atrio-femoral bypass.
\end{abstract}

This report describes our experience with this condition at one hospital over a five-year period and includes those patients who died within a few hours of admission and those who survived long enough for the diagnosis to be established and for attempted surgical repair to be undertaken. Of seven patients, two ultimately recovered.

\section{CASE REPORTS}

CASE 1 A man aged 62 was admitted to Bristol Royal Infirmary on 27 July 1963 . He was working on the supporting chains of Clifton Suspension Bridge from which he fell $20 \mathrm{ft}$. $(6 \mathrm{~m}$.) to the roadway of the bridge, apparently falling on his outstretched hands in a face-down position. He was admitted in a very shocked condition and despite attempts at resuscitation he died 90 minutes later. At necropsy he had an almost complete circumferential tear of the thoracic aorta just above the diaphragm. Other injuries included multiple fractures, among which were bilateral Colles' fractures.

CASE 2 A man aged 28 was admitted to Bristol Royal Infirmary on 4 December 1965 having been involved in a motor vehicle accident. He had sustained concussion, a fractured pelvis, fractures of the left ribs 2-10 and of the shaft of the left femur, and a ruptured diaphragm with herniation of some abdominal contents into the thorax (Fig. 1).

Operation A left postero-lateral thoracotomy through the eighth intercostal space was carried out. The left pleural cavity contained stomach, omentum, transverse colon, and spleen, together with $200-300 \mathrm{ml}$. of blood. There was bruising of the hilum of the lung but no other abnormality was detected. The left diaphragm was torn from the chest wall radially to the oesophageal hiatus. The abdominal viscera were replaced and the diaphragm was repaired with two rows of thread sutures. A Steinmann pin was inserted into the left tibial tubercle for traction of the fractured femur. At the end of the operation the patient's breathing was inadequate and tracheostomy was performed to conduct positive pressure ventilation. Postoperatively he appeared well, although he was anuric and was therefore transferred to Ham Green Hospital, the regional dialysis centre, for treatment. Haemodialysis was undertaken on two occasions but on the ninth post-operative day he died suddenly.

Necropsy findings The significant finding at necropsy was a complete circumferential tear of the aorta just below the origin of the left subclavian artery. Blood had been contained within a small sac composed of adventitia which had ruptured shortly before death, with resulting haemorrhage into the mediastinum and into the root of the neck in front of the vertebral column.

Subsequent examination of chest radiographs taken at the time of admission to Bristol Royal Infirmary and to Ham Green Hospital (the last being one day before death) showed the mediastinal shadow to be enlarged, and although none of these radiographs was truly antero-posterior, owing to the difficulty of examining this patient, the diagnosis could have been suspected earlier, with the possibility of successful treatment.

CASE 3 An 18-year-old girl was admitted on 9 July 1965 to Bristol Royal Infirmary. She had been driving a motor scooter with a pillion passenger (case 4) and had collided head-on with a saloon car. On admission she was unconscious and barely breathing and died 15 minutes later. Necropsy showed a complete circumferential tear of the aorta just beyond the left subclavian artery. There was also a fractured left tibia and fibula.

CASE 4 A young man aged 20 was admitted at the same time as case 3 , also unconscious, and he also 




FIG. 1. Case 2. Radiograph at time of admission. Evidence of ruptured left leaf of diaphragm was obvious but the mediastinal shadow was regarded as normal.

died shortly after admission. Necropsy showed an aortic tear identical to that found in case 3. There was also a fractured shaft of the right femur and multiple fractures of the pelvis.

CASE 5 A 40-year-old man was admitted to Bristol Royal Infirmary on 25 October 1966 following a road traffic accident. On examination he had surgical emphysema of the right side of the chest and an injury to the right femur. Chest radiographs, retrospectively, showed the mediastinum to be widened and there were multiple right rib fractures (Fig. 2). A radiograph of the right femur showed a supracondylar fracture. The patient was transferred to the operating theatre for suture of a lacerated scalp and insertion of a Steinmann's pin through the right tibial tubercle. At the completion of this operation, cardiac arrest supervened but he was resuscitated by external cardiac massage. Three hours later his condition deteriorated and a further chest radiograph showed the left chest to be opaque. At this stage advice was sought from the thoracic service and the patient was removed to the operating theatre where immediate left thoracotomy was undertaken. However, as soon as the chest was opened it was obvious that the aorta had ruptured and death took place almost at once. At necropsy the aorta was found to be com pletely ruptured through all layers circumferentiall at the level of the ligamentum arteriosum.

CASE 6 A 54-year-old man was admitted on $2 \&$ October 1967 having been involved in a head-on collis sion whilst in the front passenger seat of a small car. $\mathrm{He}_{\theta}$ was concussed and suffered a central dislocation of the right hip. A chest radiograph (Fig. 3) showed the mediastinal shadow to be rather wide. This patient. had undergone thoracic surgery for the repair of 8 hiatus hernia 10 years previously and fortunately there was available a follow-up radiograph takef three months previously. In comparison with the pred sent radiograph the increase in size of the mediastinat shadow was obvious (Fig. 3). An aortogram (Fig. showed an almost complete circumferential tear of the aorta just below the left subclavian artery.

Operation The patient was positioned for lef thoracotomy and the left femoral artery was exposect The left chest was opened widely through the fourth interspace. There were a few pleural adhesions resulg ing from a previous hiatus hernia repair. The aorta at and below the left sub-clavian artery, was the site of a dense haematoma. Cannulation for left atrio 


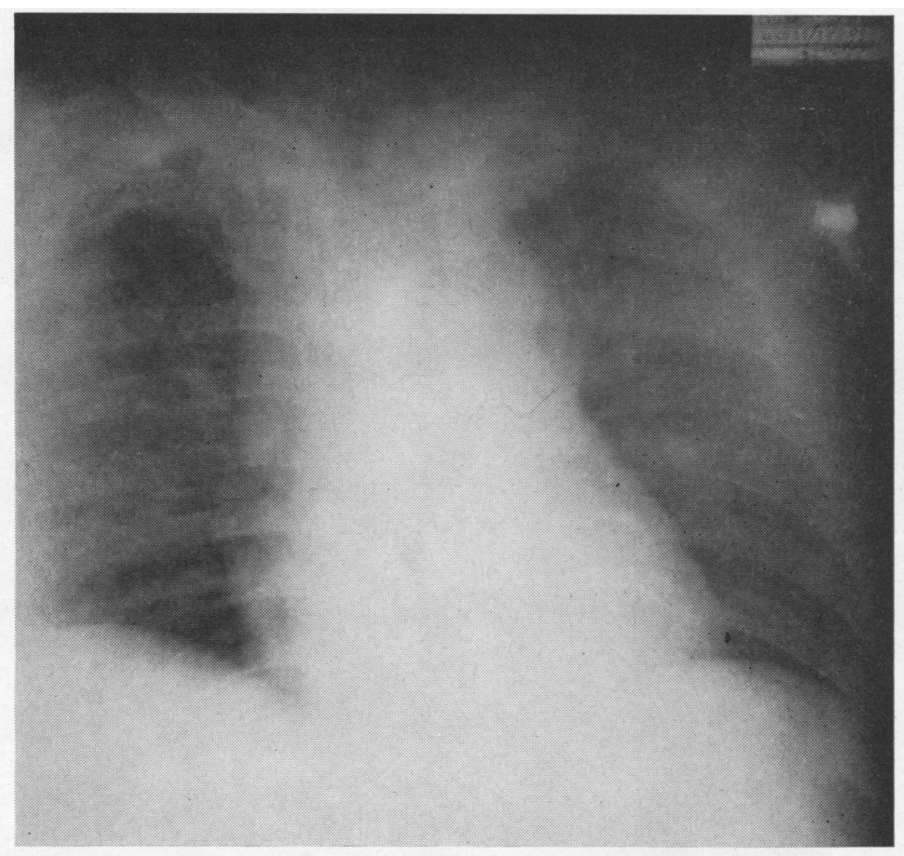

FIG. 2. Case 5. Chest radiograph at time of admission (a), with rapid progress to left haemothorax (b).

(a)

(b)

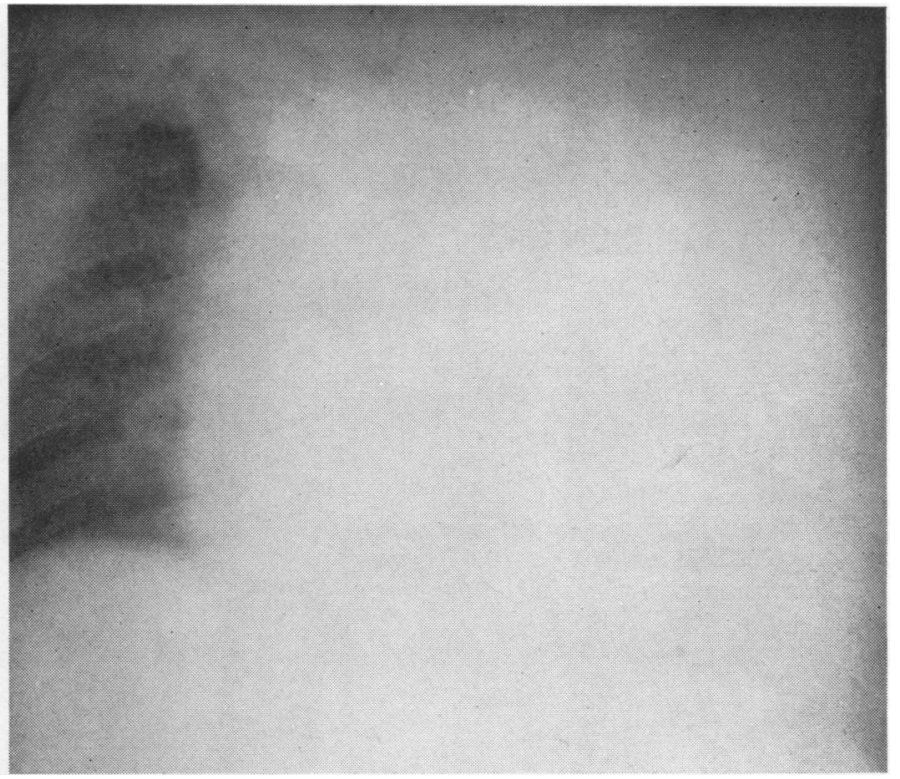

femoral bypass was undertaken before further dissection. The aorta was mobilized proximal to the left subclavian artery where it was clamped and the left subclavian artery was also clamped. The aorta was mobilized well below the haematoma and clamped, and the patient was then put on left atrio-femoral bypass using a cannula from the left atrium to a reservoir and thence to the pump and left femoral artery. It was found that a flow of 2.51 . per minute through the femoral artery allowed good perfusion and gave a right radial arterial pressure of $80 \mathrm{~mm}$. Hg. The patient passed urine during perfusion, indicating satisfactory renal perfusion. Heparin, $1 \mathrm{mg} . / \mathrm{kg}$. body weight, had been given just prior to perfusion. 




(a)

FIG. 3. Case 6. Comparison between radiograph (a) (23.5.67) and (b) (25.10.67) shows an obvious increase in the size of the aortic knuckle.

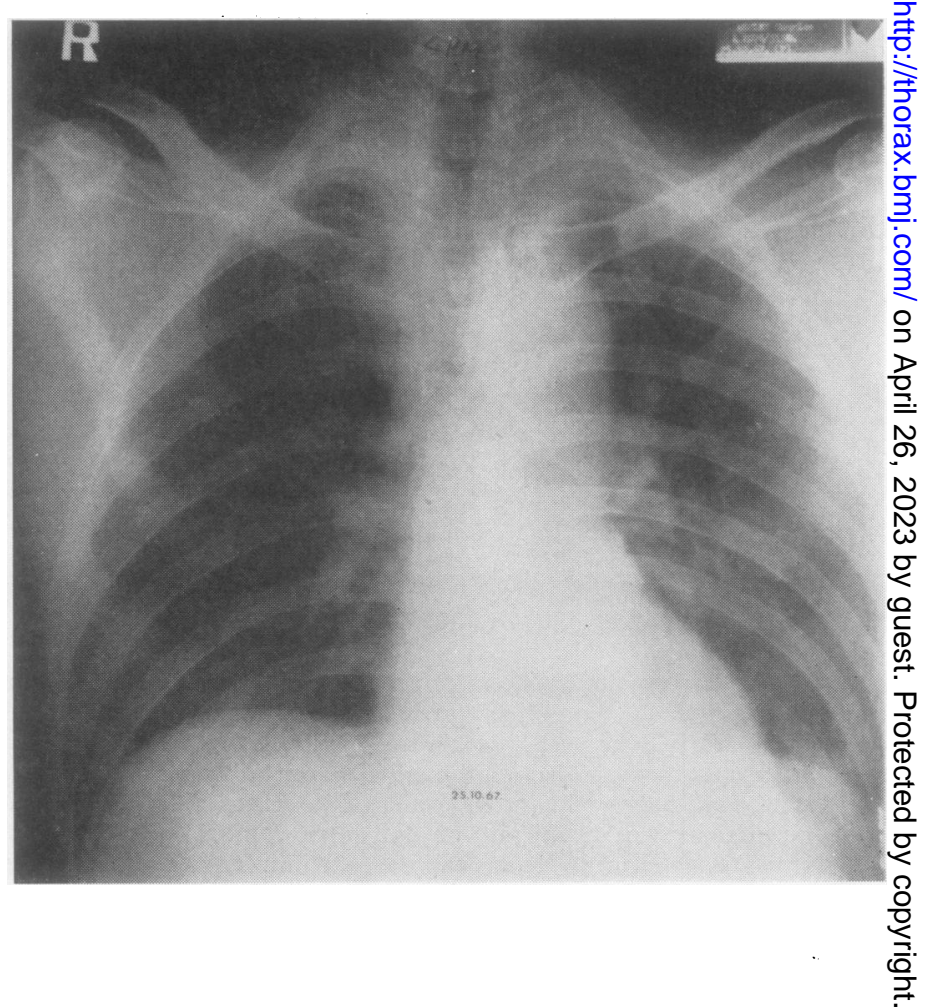




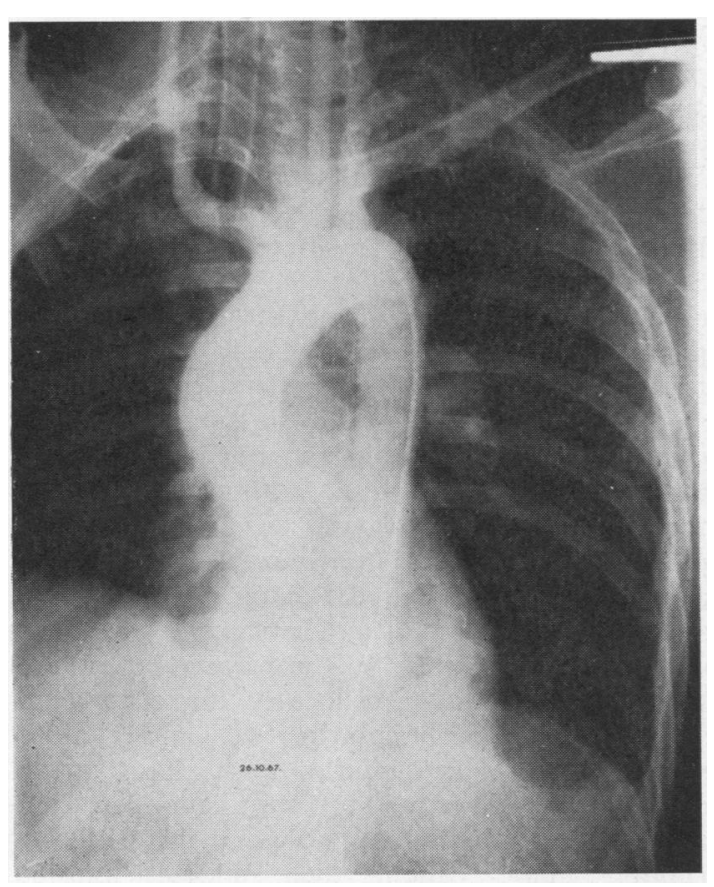

FIG. 4. Case 6. Forward aortogram demonstrating tear of aorta distal to origin of left subclavian artery.

The aortic haematoma was explored, showing an almost complete rupture of the media and a complete rupture of the intima $1 \mathrm{~cm}$. below the left subclavian artery. This area was excised and replaced with a 1 in. length of woven Dacron, using continuous sutures of $4 / 0$ silk. Following this, when the clamps were released, small bleeding points at the anastomosis were controlled with extra sutures. Heparin was neutralized with protamine sulphate, $1 \mathrm{mg} . / \mathrm{kg}$. body weight. The patient was taken off bypass and the chest was closed in layers with one pleural drain. The left femoral artery was repaired in continuity.

Post-operatively the patient made a satisfactory recovery and at the time of discharge his residual symptom was diplopia when looking to the extreme right, which was thought to be due to an associated orbital injury.

CASE 7 A 60-year-old man was admitted on 8 November 1967 having been knocked down by a car whilst road sweeping. His injuries included a laceration of the scalp, supracondylar fracture of the left femur, and a ruptured diaphragm.

Operation A left postero-lateral thoracotomy was done through the seventh intercostal space. The stomach and spleen had herniated through a rent in the diaphragm which extended radially from the chest wall almost to the oesophageal hiatus. The spleen was torn and bleeding. Splenectomy was done; the stomach was reduced into the abdomen and the diaphragm was repaired with two rows of continuous silk. In view of our previous recent experience, the aorta was examined from the subclavian artery distally, but apart from tortuosity no evidence of injury was present. Skin traction was applied to the left tibia and primary grafting of a skin defect over the vault of the skull was carried out. The patient made a good recovery from this operation. However, four days post-operatively a chest radiograph showed widening of the mediastinal shadow and an aortogram taken on the same day showed an obvious tear with dissection just distal to the left subclavian artery. Operation was undertaken three hours later.

Operation The left femoral artery was exposed and a left thoracotomy was undertaken through the fourth interspace. The left atrium was cannulated and a left atrio-femoral bypass was held in readiness. The lung was then reflected forward and the aorta was examined. It appeared to be of normal size; but there was a bruise of the whole circumference about $12 \mathrm{~cm}$. below the left subclavian artery. When the aorta was palpated more carefully, a haematoma was felt at its posterior aspect. A left atrio-femoral bypass was managed as in case 6 . A clamp was applied to the arch of the aorta just proximal to the left subclavian artery. The left subclavian artery was clamped, as was the distal aorta. The aorta was transected at the level of the left subclavian artery. One centimetre proximal to this there was a complete circumferential tear of the intima and media, extending to the lower aortic segment which appeared as two concentric tubes separated by fresh clot. The tear had also extended proximally along the aortic arch beyond the aortic clamp. The intima and media were sutured back to the adventitia using numerous $4 / 0$ silk sutures. The distally transected aorta was first repaired with a running suture re-uniting the dissected media to the adventitia, and the aorta was then repaired end-to-end, using continuous $4 / 0$ silk. The aorta was very atherosclerotic but on release of the clamps good haemostasis had been secured. The chest was closed in layers with one pleural drain, and the left femoral artery was repaired in continuity.

The patient made a slow but satisfactory recovery from his injuries.

\section{DISCUSSION}

INCIDENCE Traumatic rupture of the aorta is not rare and there are numerous reports of small groups of patients. Strassmann (1947) reported 7,000 necropsies on patients who were either dead on admission or died on the day of admission to hospital. In 69 of these $(1 \%)$ death was due to traumatic rupture of the aorta. National statistics relating to the frequency of this condition are not available in the United Kingdom. However, Brown (1967, personal communication) describes 
19 patients in whom this injury was identified at coroner's necropsies conducted by him in Bristol over the previous five years. These, together with the patients discussed in this paper, show an incidence of 26 such cases occurring in a population of about half a million in five years. Since all patients suffering a traumatic death are subject to necropsy, it is likely that these figures are substantially correct.

PATHOLOGY Rupture of the aorta involves the intima and media and is usually a circumferential tear at or just below the origin of the left subclavian artery, although avulsion of the innominate artery occurs less frequently. The haematoma is usually contained by the thick adventitia, although the ruptured ends of the media may be several centimetres apart. This false aneurysm may rupture within minutes, hours or days, or proceed to the formation of a chronic aneurysm which may remain unsuspected for many years. Rice and Wittstruck (1951) discuss the mechanism of rupture with deceleration injuries and observe that, whenever one part of the body is decelerated at a rate which differs from that of another part, the connexion between these two parts is under stress, which is proportional to the rate of deceleration. Violent deceleration, such as accompanies head-on automobile collisions, aircraft crashes or falls from a height will produce such a state of affairs, the descending thoracic aorta continuing a forward movement, whereas the aortic arch is retained by the great vessels. This probably explains the frequency of tears at the level of the ligamentum arteriosum or at the origin of the innominate artery. The aorta need not be diseased (as it was in case 7) for the majority of recorded cases occurred in young and previously healthy adults. Jahnke, Fisher, and Jones (1964) recorded six patients successfully treated at the Walter Reed General Hospital, Washington by surgical repair, using left atriofemoral bypass or cardiopulmonary bypass. These patients probably underwent a process of natural selection as they were operated on at intervals of 22 hours, $4,7,6,14$, and 15 days following injury, usually having been transferred great distancesin some cases from Europe-for surgery. Spencer, Guerin, Blake, and Bahnson (1961) reported seven such patients, one of whom survived surgery. They also reported eight cases of late traumatic aneurysm at the level of the ligamentum arteriosum, seven of whom survived resection of the aneurysm. One of this group had his operation 37 years following the presumptive injury (having been kicked by a calf). This may imply that once a $\stackrel{\text { ? }}{\rightarrow}$ patient who has developed a false aneurysm has survived for more than a few years, the ultimate $\frac{\bar{\sigma}}{\overline{0}}$ prognosis may be relatively good. Both these $\frac{\sigma}{\vec{\nabla}}$ authors stressed the value of forward aortography $\unrhd$ to locate the site of the aortic lesion. Eiseman and $\%$ Rainer (1958), Alley, Van Mierop, Li, Kausel, and $\vec{\circ}$ Stranahan (1961), Bromley, Hobbs, and Robinson (1965), and Roberts and Lord (1967) also reported $\vec{\omega}$ patients who survived surgical repair of traumatic rupture of the aorta. Most patients in these series, $\vec{x}$ and our own patients, were the victims of severe deceleration or acceleration injuries, prominent $\overrightarrow{.}$ among which were head-on automobile collisions, जे the patient usually having occupied the front 0 passenger seat. Associated injuries were frequent and included ruptured diaphragm, ruptured $\vec{c}$ spleen, fractured femur and broken ribs, occurring separately or together. In the present series, fractured femur, tibia or dislocated hip occurred in six, ruptured diaphragm in two, and ruptured $\vec{\mathscr{}}$ spleen in one. Head injuries were almost invariable. It was therefore not surprising that these associated injuries usually obscured the aortic disruption; many patients died suddenly and unexpectedly in hospital days or weeks following successful surgical treatment for other injuries $\stackrel{\square}{\mathbb{Q}}$ (case 2). This injury is more likely to be diagnosed $\stackrel{2}{\Rightarrow}$ if it is suspected, and serial radiology of the chest will often show a progressively widening medias- $\frac{3}{3}$ tinum. Suspicion is best confirmed by forward aortography, achieved by advancing a catheter (introduced by the Seldinger technique into the 으 femoral artery) into the ascending aorta, at which $\underset{x}{0}$ point the injection should be made. This seems $\dot{\sigma}$ to be the only certain way of demonstrating a break at any point in the aortic arch. This investigation was helpful in our two surgically treated cases and there is little doubt that in case 7, in 0 whom the aorta appeared normal at operation, only incontrovertible aortographic evidence of 0 aortic disruption prevented the operation being abandoned as a false alarm. Others have com- $\sigma$ mented on the frequent benign appearance of the $N$ aorta at operation. Eiseman and Rainer (1.958) reported such a patient who, despite a clinical 0 diagnosis of traumatic rupture of the aorta, was $\infty$ found to have an apparently normal aorta at $\frac{D}{\Phi}$ exploratory thoracotomy. Aortotomy was there- $\stackrel{?}{?}$ fore not carried out. However, this patient 0 developed an aneurysm, clearly seen on chest radiographs three weeks later. At a further opera- $\stackrel{\mathbb{Q}}{\stackrel{Q}{\Phi}}$

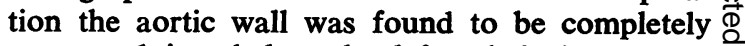
transected just below the left subclavian artery, with a $6 \mathrm{~cm}$. separation of aortic ends opening 
into the aneurysmal sac. In the patient who died of aortic rupture 9 days after thoracotomy to repair the diaphragm (case 2 ), the aorta appeared normal at operation as it also did in the first operation on the patient (case 7). The usual course is for traumatic false aneurysm to rupture in a matter of hours, days or weeks following injury. Since there is no way to determine which patients will survive to form a chronic aneurysm, an attempt at surgical repair must follow diagnosis as soon as possible and certainly in many of the patients in whom surgical repair is undertaken a successful outcome can be expected.

Left atrio-femoral bypass (Cooley, DeBakey, and Morris, 1957 ; Gerbode, Braimbridge, Osborn, Hood, and French, 1957) allows satisfactory and safe operating conditions, preventing proximal hypertension and left ventricular strain during aortic cross clamping and furthermore ensures adequate renal and spinal cord perfusion. Heparinization and its reversal by protamine poses no undue bleeding problems at the aortic repair. However, should intra-abdominal bleeding have been overlooked or intra-cerebral bleeding be taking place, heparinization might significantly affect this.
We are grateful to Dr. Rhys-Davies for performing aortography on patients 6 and 7, and to Miss A. Pakington who managed the left atrio-ventricular bypass. We also thank Dr. G. Burton and Dr. P. Baskett who anaesthetized patients 6 and 7 respectively, and Professor T. Hewer and Dr. N. Brown for access to necropsy reports on the fatal cases.

\section{REFERENCES}

Alley, R. D., Van Mierop, L. H. S., Li, E. Y., Kausel, H. W., and Stranahan, A. (1961). Traumatic aortic aneurysm: graftless excision, anastomosis. Arch. Surg., 83, 300.

Bromley, L. L., Hobbs, J. T., and Robinson, R. E. (1965). Early repair of traumatic rupture of the thoracic aorta. Brit. med. J., 2, 17.

Cooley, D. A., DeBakey, M. E. and Morris, G. C. (1957). Controlled extracorporeal circulation in surgical treatment of aortic aneurysm. Ann. Surg., 146, 473.

Gerbode, F., Braimbridge, M., Osborn, J. J., Hood, M., and French, S. (1957). Traumatic thoracic aneurysms: treatment by resection and grafting with the use of an extracorporeal bypass. Surgery, 42, 975 .

Eiseman, B., and Rainer, W. G. (1958). Clinical management of posttraumatic rupture of the thoracic aorta. J. thorac. cardiovasc. Surg., 35, 347.

Jahnke, E. J., Fisher, G. W., and Jones, R. C. (1964). Acute traumatic rupture of the thoracic aorta. Report of six consecutive cases of successful early repair. Ibid., 48, 63 .

Rice, W. G., and Wittstruck, K. P. (1951). Acute hypertension and delayed traumatic rupture of the aorta. J. Amer. med. Ass., 147, 915.

Roberts, J. C., and Lord, P. W. (1967). Ruptured thoracic aorta. Anaesthesia, 22, 415 .

Spencer, F. C., Guerin, P. F., Blake, H. A., and Bahnson, H. T. (1961). A report of 15 patients with traumatic rupture of the thoracic aorta. J. thorac. cardiovasc. Surg. 41, 1.

Strassmann, G. (1947). Traumatic rupture of the aorta. Amer. Heart J., 33, 508 . 